\title{
The JET Upgraded Toroidal Alfvén Eigenmode Diagnostic System
}

\author{
S. Dowson ${ }^{1}$, S. Dorling ${ }^{1}$, H. K. Sheikh ${ }^{1}$, T. Blackman ${ }^{1}$, G. Jones ${ }^{1}$, A. Goodyear ${ }^{1}$, P. Puglia ${ }^{2}$, \\ P. Blanchard ${ }^{2}$, A. Fasoli ${ }^{2}$, D. Testa ${ }^{2}$, N. Fil ${ }^{3}$, V. Aslanyan ${ }^{3}$, M. Porkolab ${ }^{3}$, W. Pires De Sa ${ }^{4}$, \\ R. Galvao ${ }^{4}$, L. Ruchko ${ }^{4}$, J. Figueiredo ${ }^{5,6}$, C. Perez Von Thun ${ }^{5}$, JET Contributors ${ }^{7}$ \\ EUROfusion Consortium, JET, Culham Science Centre, Abingdon, OX14 3DB, UK
}

\author{
${ }^{1}$ CCFE, Culham Science Centre, Abingdon, OX14 3DB, UK $\quad{ }^{2}$ EPFL Swiss Plasma Center, Lausanne, Switzerland \\ ${ }^{3}$ MIT PSFC, 175 Albany Street, Cambridge, MA 02139, US \\ ${ }^{4}$ Instituto de Física, Universidade de São Paulo, São Paulo CEP 05508-090; Brazil ${ }^{5}$ EUROfusion PMU, Culham \\ Science Centre, Abingdon, Oxon, OX 14 3DB United Kingdom \\ ${ }^{6}$ Instituto de Plasmas e Fusão Nuclear, Instituto Superior Técnico, Universidade de Lisboa, Portugal \\ ${ }^{7}$ See the author list of X. Litaudon et al., Nucl. Fusion 57, 102001 (2017).
}

The Alfvén Eigenmode Active Diagnostic system (AEAD) has undergone a major upgrade and redesign to provide a state-of-the-art excitation and real-time detection system for JET.

The new system consists of individual $4 \mathrm{~kW}$ amplifiers allowing for increased current, separate excitation and real time control of relative phasing between antenna currents. The amplifiers have a frequency range of $10-1000 \mathrm{kHz}$, divided into various frequency bands by external matching filters. Due to the varying transmission line impedance throughout the frequency range, the amplifiers were designed with a very high resilience to reflected power.

The existing amplifier control electronics have been replaced with a digital control system incorporating a National Instruments platform and Field Programmable Gate Array (FPGA) modules for frequency, gain and phase control with a frequency and phase resolution of less than $1 \mathrm{kHz}$ and 1 degree respectively. Complementing the digital control system is the Protection and Control System, which utilizes Field Programmable Analog Arrays (FPAAs) and an array of electronic devices to monitor and control the AEAD.

New capabilities such as independent antenna current/phase control, allow for improved excitation control, better definition of antenna spectrum combined with enhanced system reliability. This paper will review the new AEAD system, its unique capabilities and improvements over the previous diagnostic system.

Keywords: JET, TAE, FPGA, FPAA, AEAD, RF Amplifier.

\section{Introduction}

The JET Alfvén Eigenmode Active Diagnostic (AEAD) probes the background Alfvén Eigenmodes (AEs) spectra of the plasma [1]. It does so by resonantly exciting AEs [2,3] via two antenna modules, with each module housing four excitation coils. The Antenna modules are separated by $180^{\circ}$ toroidally.

Previously the excitation coils were driven by a single, high power broadband amplifier, with a maximum power output of $5 \mathrm{~kW}$ and a frequency band of $10-500 \mathrm{kHz}$. The previous system successfully measured damping rates for modes $n \leq 7$ though unfortunately struggled to measure damping rates for modes $n \geq 8$ [4-6]. Two factors strongly influenced the difficulty in exciting and characterising high-n modes: (1) These modes have a quasi-degenerate spectrum and radial eigenfunctions peaked towards the centre of the plasma column, i.e. away from the antennae. (2) Severely restricted capability to optimize the antenna phase currents (only 0 and $\pi$ possible) to excite certain $n$ modes.

To overcome these restrictions and allow for the probing of AEs in plasma configurations that were not possible in the past, a new upgraded system was proposed in preparation for the deuterium-tritium (DT) campaign. Each excitation coil is now driven by a single $4 \mathrm{~kW}$ amplifier; allowing for increased current, separate excitation and real time control of relative phasing between antenna currents with an increased frequency range of $10-1000 \mathrm{kHz}$.

In this paper, section 2 looks at the new AEAD system in-depth, with a strong focus on the new elements of the system. Section 3 reviews the operation of the AEAD system and its performance thus far.

\section{AEAD upgrade}

The system upgrade essentially consists of individual RF amplifiers driving the in-vessel excitation coils. The $\mathrm{RF}$ amplifiers in turn are driven and controlled by what is known as the Master Driver (MD) which is essentially a National Instruments (NI) PXI express chassis incorporating LabView Real Time (RT) and Field Programmable Gate Array (FPGA) software.

The total system power has increased from $5 \mathrm{~kW}$ to 24 $\mathrm{kW}(4 \mathrm{~kW} /$ Amplifier - at present six amplifiers installed driving six excitation coils), with the bandwidth being extended to $1000 \mathrm{kHz}$ to allow for MHD spectroscopy using global Alfvén waves below the continuum [7].

A schematic overview of the new system is shown in Fig. 1. 


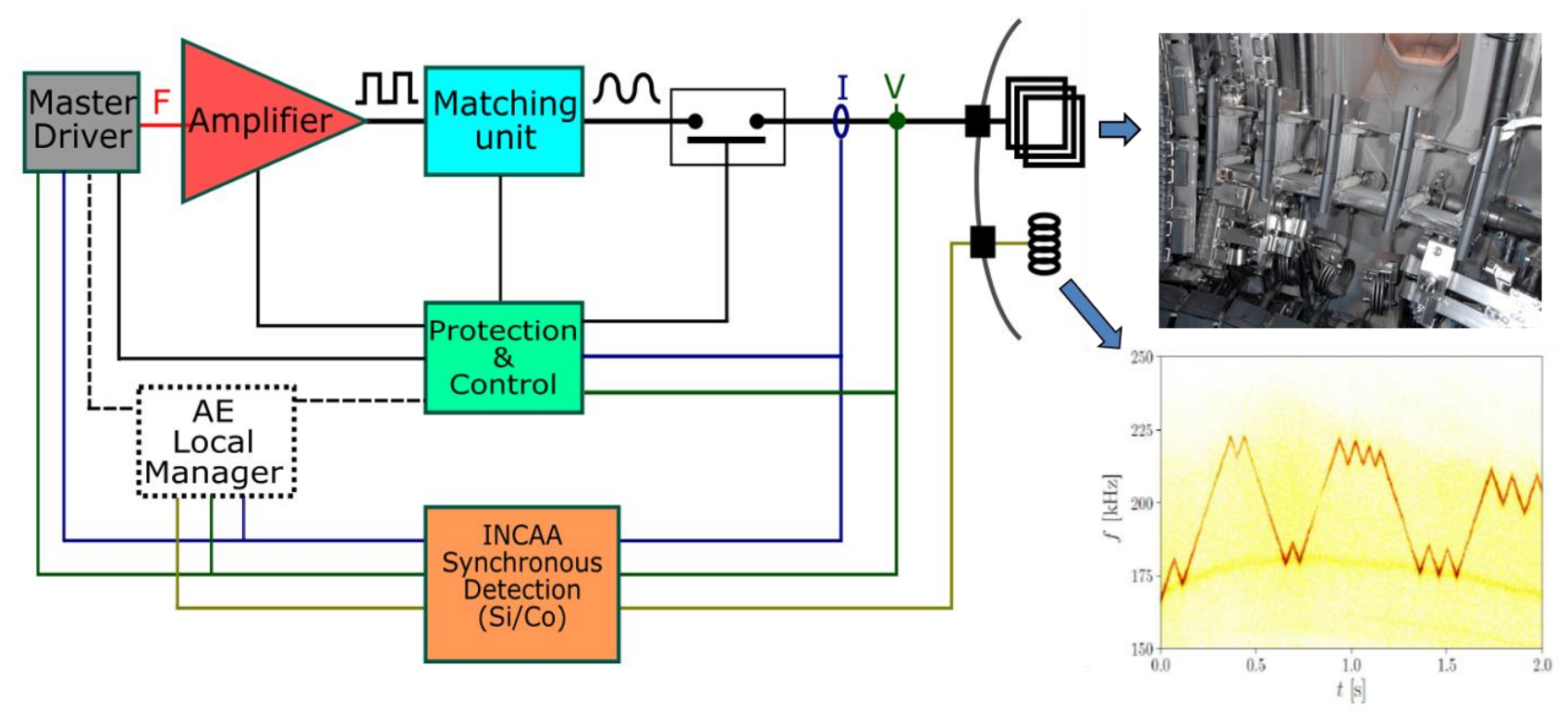

Fig. 1. A schematic overview of the AEAD system. Antenna (top right) and spectrogram showing tracking of AEs (bottom right). The INCAA synchronous detection system applies a frequency mixer with the synchronous in-phase (I) and quadrature (Q) components of the antenna signal to the incoming differential signals which are then filtered by a low-pass filter with a $<100 \mathrm{~Hz}$ bandwidth, generating their corresponding cosine (I) and sine (Q) DC components that are used by both the MD and the AE local manager for phase and current measurement as well as phase and current control. The MD drives the RF amplifiers with a small, frequency swept square-wave signal, which in turn is amplified by the amplifiers. The matching units (low pass filters) then smooth out the amplifier output, resulting in a sinusoidal wave which is then transmitted via co-axial cables (approx. 85m in length) to the antennas. The protection and control block is at the centre of the system, interconnecting with all system elements and responsible for trip management and protection of the AEAD system as well as timing and signal processing.

\subsection{RF Amplifiers and Filters}

The RF amplifier is based upon a metal-oxidesemiconductor field-effect transistor (MOSFET) switching/push-pull scheme (Fig. 2.) classified as a class$\mathrm{D}$ amplifier, with a bandwidth of $10-1000 \mathrm{kHz}$. Due to the varying transmission line impedance throughout the frequency range, the amplifiers were designed with a very high resilience to reflected power with a Voltage Standing Wave Ratio VSWR $>>10: 1$. This allows for fast frequency sweeping without tripping the amplifiers, resulting in a reliable, robust system free of nuisance tripping.

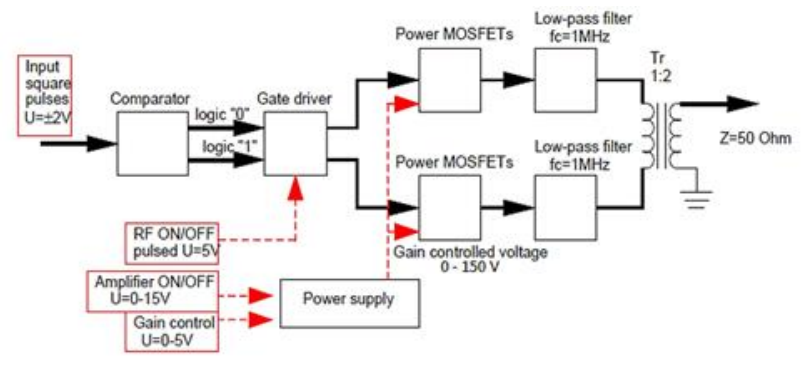

Fig. 2 Block diagram of RF circuit

The frequency range of $10-1000 \mathrm{kHz}$ is divided into various frequency bands by external matching filters. The matching filters are $18^{\text {th }}$ order, Chebyshev low pass filters that convert/smooth out the amplifier square-wave output to a sinusoidal wave and attenuate (by at least $70 \mathrm{~dB}$ ) the third harmonics. The filters have been designed in this way as the synchronous detection system is sensitive to third harmonics and will not operate correctly in their presence. The frequency response of the $50 \mathrm{kHz}$ filter can be seen in Fig. 3. with an attenuation of $\approx 80 d B$. Three frequency bands are currently in use: 50,150 and $250 \mathrm{kHz}$ $\left(f_{c}\right)$ with the bandwidth of each filter being $f_{c} / 2$.

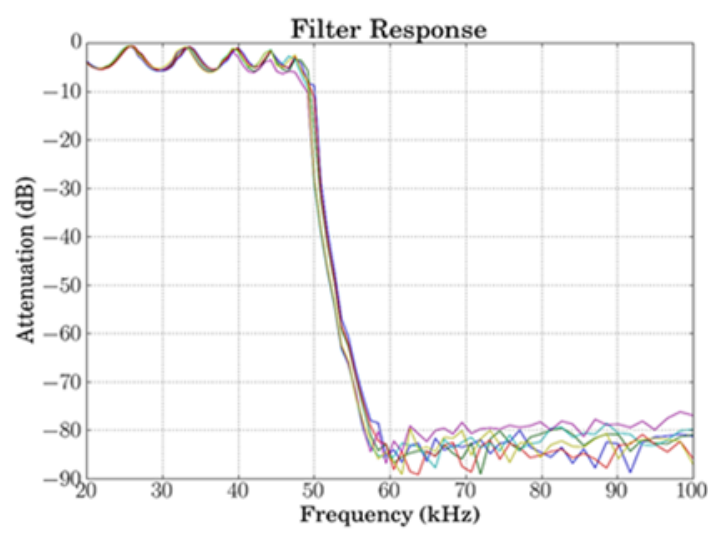

Fig. 3. Frequency response of $50 \mathrm{kHz}$ filter

\subsection{Master Driver}

The Master Driver (MD) hardware for the JET AEAD system upgrade is based on a National Instruments (NI) PXI express chassis, Model PXIe- 1075 with 18 slots. It includes an Intel i7 quad-processor embedded computer, five NI modules, and three custom signal conditioning boards fabricated at MIT. LabView Real Time (RT) and Field Programmable Gate Array (FPGA) software is implemented in the system for performing the various amplifier input and control functions, which include phase-controlled frequency swept drives to the amplifiers and amplifier gain control (Fig. 4.). The amplifier drive signals are generated in the FPGA module by a direct digital synthesis (DDS) algorithm controlled by an external swept voltage that is input to one of the DAQ channels. The FPGA board generates $1 \mathrm{GS}^{-1} \mathrm{~s}^{-1}$ of data 
points, which gives a frequency resolution of $1 \mathrm{~Hz}$ and a phase resolution of 0.3 degrees at $100 \mathrm{kHz}$.

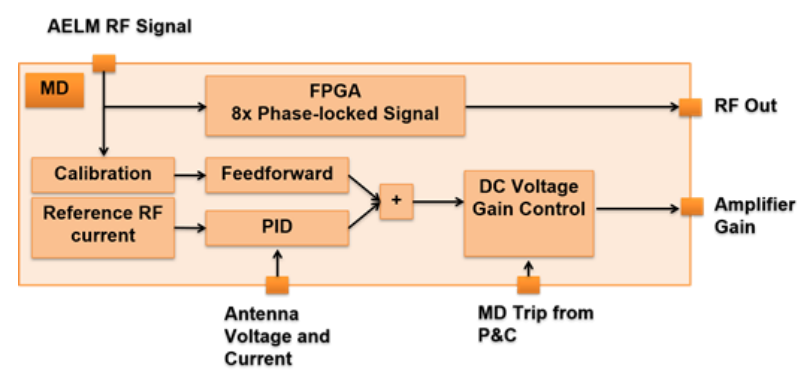

Fig. 4. Master Driver block diagram

The previous system used only one amplifier to drive all antennas allowing for only 0 and $\pi$ phase control $(+/-$ Phase). This configuration led to $n$-spectra symmetrical $n=0$ (Fig.5. LHS). Thanks to the upgrade, each RF amplifier independently excites each antenna allowing for arbitrary phase control between antenna currents and hence excitation of a particular toroidal mode number up to $|\mathrm{n}|=15$ (Fig 5. RHS).
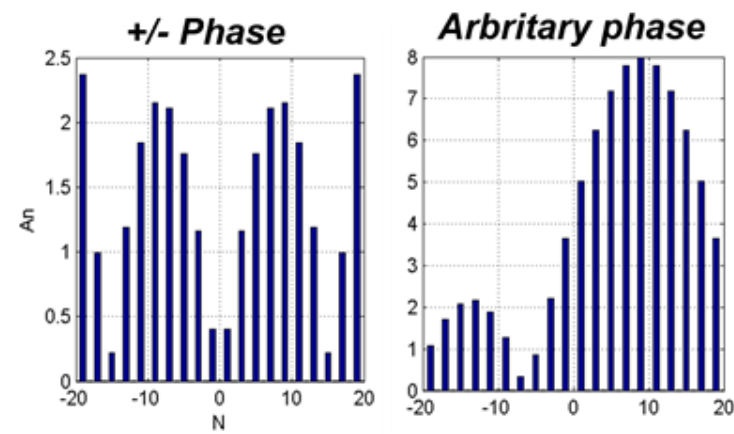

Fig. 5. Arbitrary phase optimization for specific mode excitation.

\subsection{Protection and Control}

Complementing the digital control system is the Protection and Control System which is responsible for trip management and protection of the AEAD system as well as timing and signal processing. Field Programmable Analog Arrays (FPAA) have been utilised for the signal processing and precise measurement of antenna voltage and antenna current.

The FPAA incorporates a $2 \times 2$ matrix of Configurable Analogue Blocks (CAB) surrounded by programmable interfaces and analogue input/output cells with active elements. Analogue signal processing is accomplished using an architecture based on switched capacitor circuit design. Every CAB contains two op-amps, a comparator, banks of programmable capacitors, and a collection of configurable routing and clock resources. The FPAA can be thought of as the analogue equivalent to the FPGA.

Circuit design is via Dynamx Design Lab software, a graphical design environment in which analogue signal processing blocks are connected together. Building blocks include; amplifiers, multipliers, summing blocks, integrators and other specialised bocks. Specific behaviours for each of the blocks are set by the user.

For each antenna three FPAAs are daisy-chained together for the measurement of antenna voltage, current and earth leakage current. The software for each of these measurements is identical with only the coefficients in the Look Up Table (LUT) changing (Fig. 6.)

The FPAA monitors the measured signal in and frequency reference. Due to the mismatch on the

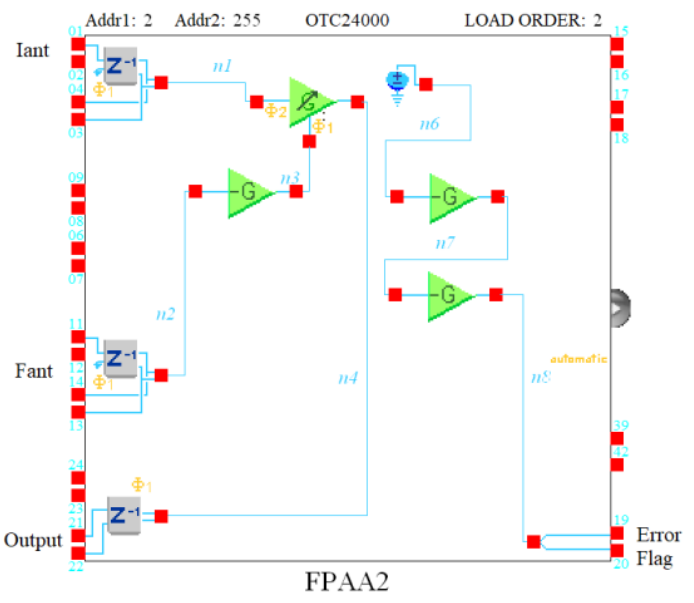

Fig. 6. Software for measurement of antenna current.

measurement lines and large frequency sweep/bandwidth of system, the output from the voltage and current measurement coils varies with frequency. The Variable Gain Amplifier (VGA) refers to the LUT and selects the correct gain coefficient according to the frequency. Two clocks are required for the multiplier $\mathrm{CAB}$, a relatively slow one for the gain stage (measured signal input -1 $\mathrm{MHz}$ ) and a second faster one, 16 times faster for the ADC (frequency reference input - $16 \mathrm{MHz}$ ). The 16:1 clock ratio required arises from the need to allow time for the $\mathrm{ADC}$ to convert the frequency input to a digital output, some nine steps. The software also monitors the internal reference voltage, which in the event of FPAA failure, will go to zero, thus producing an error flag. Fig. 8. shows the signal processing path of the measurement signals.

\section{System performance and operation}

The AEAD diagnostic has been successfully commissioned with the latest enhancements and operated up to the limits imposed by the transmission line feedthroughs on the JET vessel (1.1kV and 15A). Due to the inductance of the antennas, the maximum current that

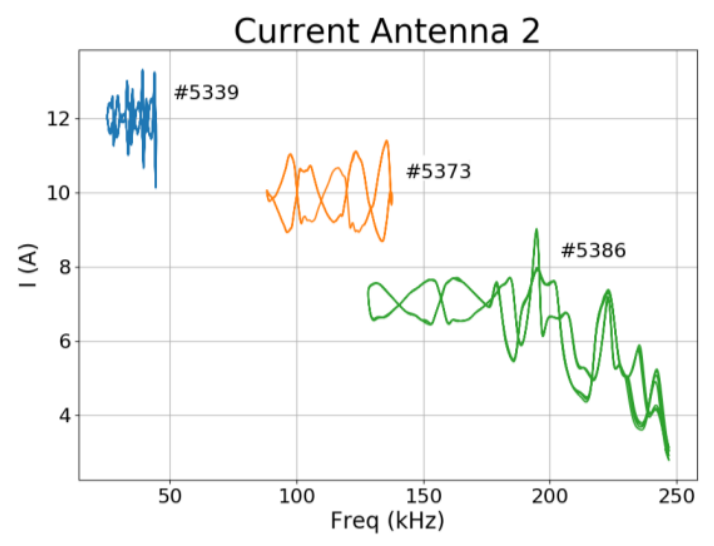

Fig. 7. Current measured in frequency bands; 50, 150 and $250 \mathrm{kHz}$. 


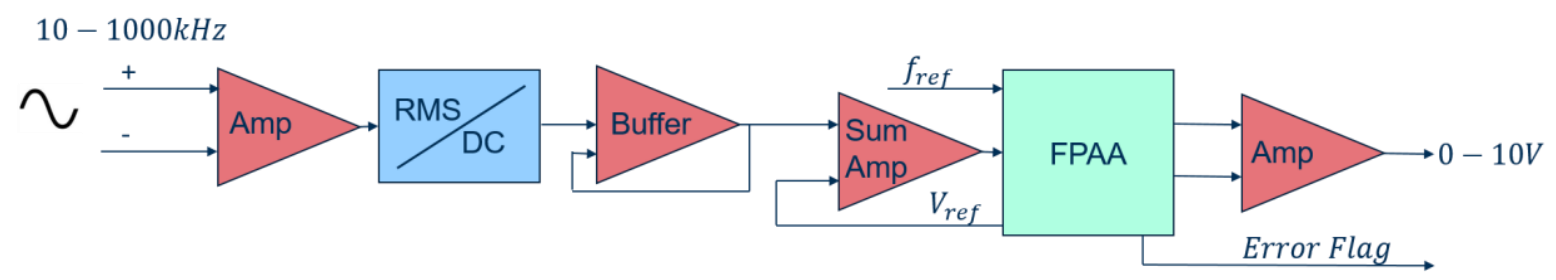

Fig. 8. Signal path of measurement signal shown. Raw signal is converted to RMS then peak value is recovered before being buffered and then summed with FPAA reference voltage. This off-set/addition is necessary to ensure the signal is in within the acceptable range for the FPAA. The final output is a $0-10 \mathrm{~V}$ signal out representing measurement signal in.

can be driven in each coil is dictated by the frequency band of operation and the feedthrough voltage limit (Fig. 7.).

Real time phase control with frequency sweeps at $200 \mathrm{kHz} / \mathrm{s}$ on various antennas has been demonstrated successfully (Fig. 10.). Small spikes can be seen in the phase control however the MD control loops are still being optimised for peak performance.

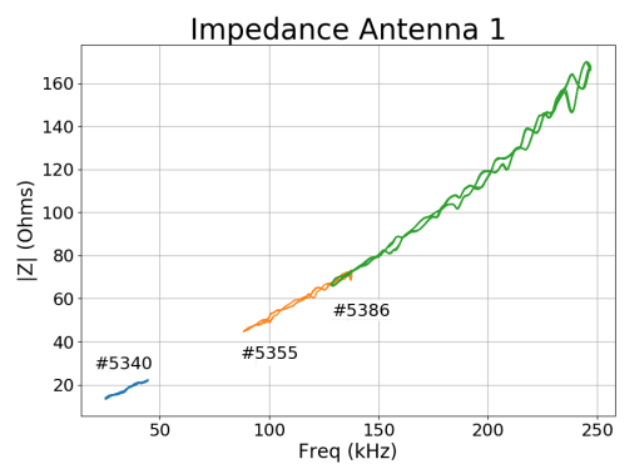

Fig. 9. Impedance calculated from Antenna voltage and currents in frequency bands 50,150 and $250 \mathrm{kHz}$.

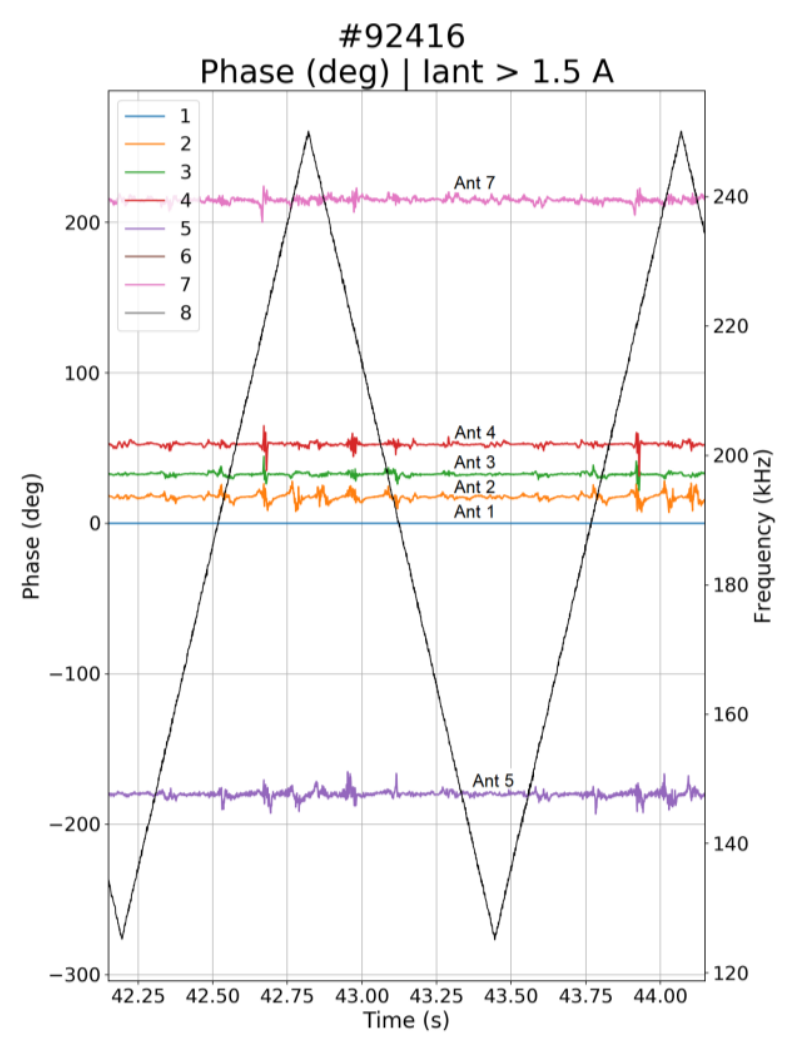

Fig. 10. Real time phase control during frequency sweep at $200 \mathrm{kHz} / \mathrm{s}$

\section{Conclusion}

In summary, the AEAD has been successfully upgraded and shown to be a robust and reliable system capable of detecting and tracking modes. Increased power $(\approx 5 \mathrm{x}$ that of previous system) and real time phase control will allow for mode number identification up to $|\mathrm{n}| \leq 15$.

Possible further work would be to implement phase two of the project; a new FPGA based digital synchronous detection system with a frequency range of $1000 \mathrm{kHz}$ and the ability to excite and track in real time simultaneously different mode numbers.

\section{Acknowledgments}

This work has been carried out within the framework of the EUROfusion Consortium and has received funding from the Euratom research and training programme 20142018 under grant agreement No. 633053. The views and opinions expressed herein do not necessarily reflect those of the European Commission'. The Brazilian group works under the scientific agreement for cooperation between the European Atomic Energy Community and the Government of the Federative Republic of Brazil in the field of fusion energy research and has been supported by the Brazilian agencies FAPESP, Project 2011/50773-0, and CNPq, Project 480733/2013-9. The work of the US collaborators at MIT was supported by the US DOE Grant DE-FG02-99ER54563. This work was also supported in part by the Swiss National Science Foundation.

\section{References}

[1] Fasoli, et al., Plasma Physis. Control. Fusion 44, B159 (2002).

[2] D. Testa, et al., Proc. 23rd SOFT, (2005) 143354.

[3] D. Testa, et al., Nucl. Fusion 50 (2010) 084010.

[4] Panis T., Fasoli A. and Testa D. 2012 Nucl. Fusion 52023013.

[5] Panis T., Fasoli A. and Testa D. 2012 Nucl. Fusion 52 023014.

[6] Testa D., Carfantan H., Goodyear A., Blanchard P., Klein A. and Panis T. 2010 Europhys. Lett. 9250001.

[7] Puglia P.G.P.P., Elfimov A.G., Ruchko L.F., Galvão R.M.O., Guimarães-Filho Z., Ronchi G. and TCABR Team 2015 Phys. Plasmas 21122509 